\title{
ON ZONOTOPES
}

\author{
BY \\ P. MCMULLEN
}

\begin{abstract}
In this paper is described a diagram technique for zonotopes, or vector sums of line segments, which is analogous to that of Gale diagrams for general polytopes, and central diagrams for centrally symmetric polytopes. The use of these new zonal diagrams leads to relationships between zonotopes with $n$ zones of dimensions $d$ and $n-d$, and enables one to enumerate all the combinatorial types of $d$ zonotopes with $n \leqq d+2$ zones. The connexion between arrangements of hyperplanes in projective space and zonotopes leads to corresponding new results about arrangements.
\end{abstract}

1. Introduction. Several recent advances in the theory of convex polytopes have stemmed from the idea of Gale diagrams, which were first conceived by Gale [1956], and later much elaborated by Perles (see Grünbaum [1967, 5.4, 6.3 and elsewhere]). The technique is to represent a $d$-polytope with $n$ vertices by a diagram of $n$ points in $(n-d-1)$-dimensional space, from which all the combinatorial properties of the original polytope can be determined.

However, Gale diagrams proved ineffective in dealing with the problems of centrally symmetric polytopes. McMullen-Shephard [1968] described the appropriate analogues of Gale diagrams for centrally symmetric polytopes; these diagrams are known as central diagrams or c.s. diagrams.

One subclass of the centrally symmetric polytopes, which is interesting for many reasons (in particular, the connexion with arrangements of hyperplanes, for which see $\$ 7$ below), comprises the zonotopes. Since a $d$-dimensional zonotope has at least $2^{d}$ vertices, even central diagrams fail to give much information about zonotopes. In this paper, we shall describe an appropriate diagram technique for zonotopes; the corresponding diagrams will be called zonal diagrams.

The author wishes to thank Professors B. Grünbaum and G. C. Shephard for many helpful comments on this paper. In particular, several improvements to $\S 5$ resulted from suggestions of Professor Shephard. He wishes also to thank Professor L. M. Kelly for several stimulating discussions on arrangements of hyperplanes, which considerably assisted in the writing of $\S 7$.

2. Zonotopes. A zonotope is the vector sum of a finite number of closed line segments in some Euclidean space. (For the terminology employed here and elsewhere, the reader is referred to Grünbaum [1967].) Alternatively, we may charac-

Presented to the Society, January 25, 1970; received by the editors April 23, 1970.

AMS 1969 subject classifications. Primary 5210; Secondary 5030.

Key words and phrases. Polytope, zonotope, zonal diagram, derived zonotope, arrangement of hyperplanes. 
terize a zonotope as a convex polytope, all of whose 2-faces are centrally symmetric. We shall usually suppose that no two of the line segments are parallel, and, without loss of generality, we may take the segments to be centered at the origin. So, a $d$-dimensional zonotope (or $d$-zonotope) can be written in the form

$$
P=S_{1}+\cdots+S_{n}
$$

where

$$
S_{i}=\operatorname{conv}\left\{-z_{i}, z_{i}\right\}, \quad i=1, \ldots, n,
$$

and $\operatorname{lin}\left\{z_{1}, \ldots, z_{n}\right\}=E^{d}$. A general point of $P$ is of the form

$$
p=\lambda_{1} z_{1}+\cdots+\lambda_{n} z_{n},
$$

where $\left|\lambda_{i}\right| \leqq 1(i=1, \ldots, n)$. In particular, each vertex of $P$ is of the form

$$
\varepsilon_{1} z_{1}+\cdots+\varepsilon_{n} z_{n}
$$

for some $\varepsilon_{i}= \pm 1(i=1, \ldots, n)$; it should be noticed, however, that in general not all points of this form are vertices of $P$.

A face $F$ of $P$ can be written

$$
F=S_{1 \sigma}+\cdots+S_{r \sigma}+\varepsilon_{r+1} z_{(r+1) \sigma}+\cdots+\varepsilon_{n} z_{n \sigma},
$$

for some permutation $\sigma$ of $\{1, \ldots, n\}$, and some $\varepsilon_{i}= \pm 1(i=r+1, \ldots, n)$. To see this, suppose that

$$
H=\left\{x \in E^{d} \mid\langle x, u\rangle=\alpha\right\}
$$

is the supporting hyperplane of $P$ such that $F=H \cap P$, and that

$$
P \subset H^{-}=\left\{x \in E^{d} \mid\langle x, u\rangle \leqq \alpha\right\} .
$$

Let $H_{0}$ be the hyperplane through the origin parallel to $H$; then certain of the points $z_{t}$ will lie in $H_{0}$, say $z_{1 \sigma}, \ldots, z_{r \sigma}$, and the remaining points $\pm z_{(r+1) \sigma}, \ldots, \pm z_{n \sigma}$ will lie in the open half-spaces bounded by $H_{0}$. Suppose that, for $i=r+1, \ldots, n$,

$$
\varepsilon_{i} z_{i \sigma} \in \text { int } H_{0}^{+}=\left\{x \in E^{d} \mid\langle x, u\rangle>0\right\} ;
$$

then if $z=\eta_{r+1} z_{(r+1) \sigma}+\cdots+\eta_{n} z_{n \sigma}\left(\eta_{i}= \pm 1, i=r+1, \ldots, n\right)$, we see that

$$
\langle z, u\rangle \leqq\left\langle\varepsilon_{r+1} z_{(r+1) \sigma}+\cdots+\varepsilon_{n} z_{n \sigma}, u\right\rangle,
$$

with strict inequality unless $\eta_{i}=\varepsilon_{i}(i=r+1, \ldots, n)$. Since $S_{i \sigma} \subset H_{0}(i=1, \ldots, r)$ it follows that

$$
F=S_{1 \sigma}+\cdots+S_{r \sigma}+\varepsilon_{r+1} z_{(r+1) \sigma}+\cdots+\varepsilon_{n} z_{n \sigma},
$$

as claimed above.

It should be noticed that the argument used above did not depend upon the line segments $S_{i}$ being nonparallel, and so the description of the faces applies to any vector sum of line segments. 
The description of the faces of the zonotope $P$ shows that if $H$ is any hyperplane such that $H \cap P=F$, then the normal vector $u$ to $H$ is orthogonal to each of the line segments $S_{i \sigma}(i=1, \ldots, r)$. So, to every segment $S_{i}(i=1, \ldots, n)$ with $z_{i} \neq 0$, there corresponds a zone of faces, which are the intersections with $P$ of supporting hyperplanes with normal vectors orthogonal to $z_{i}$. If no two of the segments $S_{i}$ are parallel, we shall say $P$ is a zonotope with $n$ zones.

In what follows, we may without loss of generality suppose that, after suitable relabelling and changes of sign of the points $z_{i}$, we can write any particular face $F$ in which we are interested in the form

$$
F=S_{1}+\cdots+S_{r}+z_{r+1}+\cdots+z_{n} .
$$

Any point of a polytope $P$ is a relatively interior point of exactly one face of $P$ (in this context, we define $P$ to be an improper face of itself). If the point $p=z_{1}+\cdots+z_{n}$ is a vertex of $P$, and $z_{i} \neq 0(i=1, \ldots, n)$, then we have seen that there is an open half-space of $E^{d}$ bounded by a hyperplane $H_{0}$ through 0 which contains every $z_{i}(i=1, \ldots, n)$. If $p$ is not a vertex, then suppose that $p$ is a relatively interior point of the face $F=S_{1}+\cdots+S_{r}+z_{r+1}+\cdots+z_{n}$ (included here are the cases when any or all of $z_{1}, \ldots, z_{r}$ are the zero vector). Then we can write

$$
p=\lambda_{1} z_{1}+\cdots+\lambda_{r} z_{r}+z_{r+1}+\cdots+z_{n},
$$

for some $\left|\lambda_{i}\right|<1(i=1, \ldots, r)$. Subtracting the second expression for $p$ from the first, we obtain

$$
\left(1-\lambda_{1}\right) z_{1}+\cdots+\left(1-\lambda_{r}\right) z_{r}=0
$$

and since $1-\lambda_{i}>0(i=1, \ldots, r)$, we see by dividing this expression by $\sum_{i=1}^{r}\left(1-\lambda_{i}\right)$ that

$$
0 \in \text { rel int } \operatorname{conv}\left\{z_{1}, \ldots, z_{r}\right\},
$$

the relative interior of the convex hull of $\left\{z_{1}, \ldots, z_{r}\right\}$. Conversely, if this condition holds, say

$$
0=\mu_{1} z_{1}+\cdots+\mu_{r} z_{r}
$$

for some $\mu_{i}>0(i=1, \ldots, r)$ with $\sum_{i=1}^{r} \mu_{i}=1$, then

$$
\begin{aligned}
p & =z_{1}+\cdots+z_{n} \\
& =\left(1-\mu_{1}\right) z_{1}+\cdots+\left(1-\mu_{r}\right) z_{r}+z_{r+1}+\cdots+z_{n},
\end{aligned}
$$

which, since $\left|1-\mu_{i}\right|<1(i=1, \ldots, r)$ implies that $p \in \operatorname{rel}$ int $F$.

We may divide the points $\varepsilon_{1} z_{1}+\cdots+\varepsilon_{n} z_{n}\left(\varepsilon_{i}= \pm 1, i=1, \ldots, n\right)$ into three classes: vertices, boundary points and interior points of $P$. In view of the previous discussion, we shall not call any of these points vertices if any $z_{i}=0$, since then the points coincide in sets of 2 or more, and no supporting hyperplane of $P$ can contain just one of the points. Obviously $P$ can have no such interior points if one or more 
subsets of $n-1$ of the points $z_{1}, \ldots, z_{n}$ lie in a hyperplane (in which case $P$ will be a prism).

The description of the faces of the zonotope $P$ makes it clear that, if $\Lambda$ is a nonsingular linear transformation of $E^{d}$, and if $\nu_{i}>0(i=1, \ldots, n)$, then

$$
Q=\left(\nu_{1} S_{1}+\cdots+v_{n} S_{n}\right) \Lambda
$$

is a zonotope combinatorially isomorphic to $P$. We shall say that $P$ and $Q$ are equivalent zonotopes. Of course, in general not all zonotopes $Q$ that are combinatorially isomorphic to $P$ are equivalent to $P$; if they are, we shall say that $P$ is zonally stable. We shall briefly discuss zonally stable zonotopes in $\S 8$.

Of interest also are the polytopes dual to zonotopes. Let $P^{*}$ be the polar reciprocal of the zonotope $P$; that is,

$$
P^{*}=\left\{x \in E^{d} \mid\langle x, p\rangle \leqq 1 \text { for all } p \in P\right\} .
$$

Referring to the general expression for a point of $P$, we see that

$$
x \in P^{*} \leftrightarrow \sum_{i=1}^{n} \lambda_{i}\left\langle x, z_{i}\right\rangle \leqq 1,
$$

for all $\left|\lambda_{i}\right| \leqq 1(i=1, \ldots, n)$; considering the particular values $\lambda_{i}= \pm 1(i=1, \ldots, n)$, it follows that $P^{*}$ consists of just those points $x \in E^{d}$ such that

$$
\sum_{i=1}^{n}\left|\left\langle x, z_{i}\right\rangle\right| \leqq 1
$$

The face $\hat{F}$ of $P^{*}$ corresponding to the face

$$
F=S_{1}+\cdots+S_{r}+z_{r+1}+\cdots+z_{n}
$$

of $P$ is

$$
\hat{F}=\left\{x \in P^{*} \mid\langle x, p\rangle=1 \text { for all } p \in F\right\},
$$

from which it is easy to deduce that

$$
\hat{F}=\left\{x \in P^{*} \mid\left\langle x, z_{i}\right\rangle=0(i=1, \ldots, r),\left\langle x, z_{r+1}+\cdots+z_{n}\right\rangle=1\right\} .
$$

We notice that the faces of $P^{*}$ corresponding to the faces in the $i$ th zone of $P$ (that is, to those containing a translate of $S_{i}$ ) lie in the hyperplane

$$
H_{i}=\left\{x \in E^{d} \mid\left\langle x, z_{i}\right\rangle=0\right\} .
$$

The facets (or $(d-1)$-faces) of $P^{*}$ are each bounded by a subset of the hyperplanes $H_{i}$.

3. Zonal diagrams. Let $Z=\left( \pm z_{1}, \ldots, \pm z_{n}\right)$ be a centrally symmetric set of points which spans $E^{d}$. A central representation (or c.s. transform) of $Z$ is constructed as follows. (We shall follow the treatment of McMullen-Shephard [1968], and refer the reader to that paper for fuller details.) A linear dependence of 
$Z_{+}=\left(z_{1}, \ldots, z_{n}\right)$ is a vector $\left(\alpha_{1}, \ldots, \alpha_{n}\right)$ of $E^{n}$ which satisfies

$$
\alpha_{1} z_{1}+\cdots+\alpha_{n} z_{n}=0 .
$$

It is clear that the set $L\left(Z_{+}\right)$of linear dependences of $Z_{+}$forms a linear subspace of $E^{n}$ of dimension $n-d$. Let $\left\{a_{1}, \ldots, a_{n-d}\right\}$ be a basis of $L\left(Z_{+}\right)$, say $a_{j}=\left(\alpha_{j 1}, \ldots, \alpha_{j n}\right)$ $(j=1, \ldots, n-d)$. If we write

$$
\bar{z}_{i}=\left(\alpha_{1 i}, \ldots, \alpha_{n-d, i}\right) \in E^{n-d} \quad(i=1, \ldots, n),
$$

then $\bar{Z}=\left( \pm \bar{z}_{1}, \ldots, \pm \bar{z}_{n}\right)$ is a central representation of $Z$.

Among the properties of central representations we shall need are the following. The central representation $\bar{Z}$ is defined to within nonsingular linear transformation of $E^{n-d}$, and $Z$ is a central representation of $\bar{Z}$ (from which it follows that $\bar{Z}$ is a central representation of every set linearly equivalent to $Z$ ). It is clear that if $\nu_{i} \neq 0(i=1, \ldots, n)$, then a central representation of $\left( \pm \nu_{1} z_{1}, \ldots, \pm v_{n} z_{n}\right)$ is $\left( \pm \nu_{1}^{-1} \bar{z}_{1}, \ldots, \pm \nu_{n}^{-1} \bar{z}_{n}\right)$. Finally, a central representation of the subset $\left( \pm z_{1}, \ldots, \pm z_{r}\right)$ of $Z$ is obtained by projecting the corresponding subset $\left( \pm \bar{z}_{1}, \ldots, \pm \bar{z}_{r}\right)$ of $\bar{Z}$ orthogonally on to the subspace of $E^{n-d}$ completely orthogonal to the subspace spanned by $\bar{z}_{r+1}, \ldots, \bar{z}_{n}$.

Notice that, if $a=\left(\alpha_{1}, \ldots, \alpha_{n}\right)$ is any linear dependence of $Z_{+}=\left(z_{1}, \ldots, z_{n}\right)$, then, for some $\beta_{1}, \ldots, \beta_{n-d}$,

$$
a=\beta_{1} a_{1}+\cdots+\beta_{n-d} a_{n-d},
$$

from which it follows that

$$
\alpha_{i}=\left\langle b, \bar{z}_{i}\right\rangle \quad(i=1, \ldots, n),
$$

where $b=\left(\beta_{1}, \ldots, \beta_{n-d}\right) \in E^{n-d}$. Conversely, any $b \in E^{n-d}$ gives rise to a linear dependence of $Z_{+}$in this manner. The symmetry between $Z$ and $\bar{Z}$ shows that there is a corresponding relationship between vectors in $E^{d}$ and linear dependences of $\bar{Z}_{+}=\left(\bar{z}_{1}, \ldots, \bar{z}_{n}\right)$.

A centrally symmetric set of points which spans $E^{d}$ clearly gives rise to a $d$ zonotope; our first theorem discusses the relationship between this zonotope and a central representation of the original set.

THEOREM 1. Let $Z=\left( \pm z_{1}, \ldots, \pm z_{n}\right)$ be a centrally symmetric set of points which spans $E^{d}$, let $\bar{Z}=\left( \pm \bar{z}_{1}, \ldots, \pm \bar{z}_{n}\right)$ be a central representation of $Z$, and let

$$
P=S_{1}+\cdots+S_{n}, \quad S_{i}=\operatorname{conv}\left\{-z_{i}, z_{i}\right\} \quad(i=1, \ldots, n) .
$$

Let $\sigma$ be a permutation of $\{1, \ldots, n\}$, and let $\varepsilon_{i}= \pm 1(i=r+1, \ldots, n)$. Then

$$
F=S_{1 \sigma}+\cdots+S_{r \sigma}+\varepsilon_{r+1} z_{(r+1) \sigma}+\cdots+\varepsilon_{n} r_{n \sigma}
$$

is a face of $P$ if and only if

$$
0 \in \text { rel int conv }\left\{\varepsilon_{r+1} \bar{z}_{(r+1) \sigma}, \ldots, \varepsilon_{n} \bar{z}_{n \sigma}\right\} .
$$


There is no loss in generality in supposing that $\sigma$ is the identity permutation, and that $\varepsilon_{i}=1(i=r+1, \ldots, n)$. From the description of the faces of the zonotope $P$ in $\S 2$, we see that $F$ is a face of $P$ if and only if there is a vector $u \in E^{d}$ such that

$$
\begin{array}{ll}
\left\langle u, z_{i}\right\rangle=0 & (i=1, \ldots, r), \\
\left\langle u, z_{i}\right\rangle=\mu_{i}>0 & (i=r+1, \ldots, n) .
\end{array}
$$

We may clearly choose the magnitude of $u$ so that $\sum_{i=r+1}^{n} \mu_{i}=1$. From the definition of the central representation, it follows that the vector

$$
\left(0, \ldots, 0, \mu_{r+1}, \ldots, \mu_{n}\right)
$$

is a linear dependence of $\bar{Z}_{+}=\left(\bar{z}_{1}, \ldots, \bar{z}_{n}\right)$, and so

$$
\mu_{r+1} \bar{z}_{r+1}+\cdots+\mu_{n} \bar{z}_{n}=0
$$

That is,

$$
0 \in \text { rel int conv }\left\{\bar{z}_{r+1}, \ldots, \bar{z}_{n}\right\} .
$$

The argument is clearly reversible, and this establishes the theorem.

The particular case where no two of the line segments are parallel is obviously important. If

$$
P=S_{1}+\cdots+S_{n}, \quad S_{i}=\operatorname{conv}\left\{-z_{i}, z_{i}\right\} \quad(i=1, \ldots, n),
$$

is a $d$-zonotope with $n$ zones, then a central representation $\bar{Z}$ of the set $\left( \pm z_{1}, \ldots, \pm z_{n}\right)$ will be called a zonal diagram of $P$.

It is natural to ask which centrally symmetric sets $\bar{Z}$ are zonal diagrams of zonotopes; this question is answered by the following theorem.

THEOREM 2. Let $\bar{Z}=\left( \pm \bar{z}_{1}, \ldots, \pm \bar{z}_{n}\right)$ be a centrally symmetric set of points in $E^{n-d}$. Then $\bar{Z}$ is a zonal diagram of some d-zonotope with $n$ zones if and only if every open half-space of $E^{n-d}$ bounded by a hyperplane through 0 contains at least 3 points of $\bar{Z}$.

For, let $Z=\left( \pm z_{1}, \ldots, \pm z_{n}\right)$ be a central representation of $\bar{Z}$ (so that $\bar{Z}$ is a central representation of $Z$ ), let

$$
S_{i}=\operatorname{conv}\left\{-z_{i}, z_{i}\right\} \quad(i=1, \ldots, n),
$$

and write

$$
P=S_{1}+\cdots+S_{n}
$$

Bearing in mind the relationship between linear dependences of $\left(z_{1}, \ldots, z_{n}\right)$ and vectors in $E^{n-d}$, it is immediate that the condition stated in the theorem is equivalent to the condition that there be no (nontrivial) linear relations between 2 or fewer of $\left(z_{1}, \ldots, z_{n}\right)$, that is, to the condition that no two of $S_{1}, \ldots, S_{n}$ be parallel. This proves the theorem. 
A number of results about zonal diagrams are analogous to those for Gale and central diagrams, and we shall list them here without proof. Throughout, we shall keep to the notation and conventions introduced above.

Proposition 1. $P$ is a prism with basis $S_{1}+\cdots+S_{i-1}+S_{i+1}+\cdots+S_{n}$ if and only if $\bar{z}_{i}=0$.

Let $\bar{V}=\left( \pm \bar{v}_{1}, \ldots, \pm \bar{v}_{n}\right)$ be another centrally symmetric set of $2 n$ points which spans $E^{n-d}$. We say that $\bar{Z}$ and $\bar{V}$ are isomorphic if for each choice $\left(\varepsilon_{i(1)}, \ldots, \varepsilon_{i(r)}\right)$, where $\varepsilon_{i(s)}= \pm 1(s=1, \ldots, r)$, and the subscripts $i(1), \ldots, i(r)$ are distinct, the conditions

$$
\begin{aligned}
& 0 \in \text { rel int conv }\left\{\varepsilon_{i(s)} \bar{z}_{i(s)} \mid s=1, \ldots, r\right\} \\
& 0 \in \text { rel int conv }\left\{\varepsilon_{i(s)} \bar{v}_{i(s)} \mid s=1, \ldots, r\right\}
\end{aligned}
$$

are equivalent. We say $\bar{Z}$ and $\bar{V}$ are equivalent if there are positive numbers $\bar{\nu}_{1}, \ldots, \bar{\nu}_{n}$, and a nonsingular linear transformation $\bar{\Lambda}$ of $E^{n-d}$ into itself, such that $\bar{v}_{i}=\bar{v}_{i} \bar{z}_{i} \bar{\Lambda}(i=1, \ldots, n)$. Then (subject to suitable relabelling of the points of the zonal diagrams, and changes of sign) we have

Proposition 2. Two d-zonotopes with $n$ zones are combinatorially isomorphic if and only if their zonal diagrams are isomorphic.

Proposition 3. Two d-zonotopes with $n$ zones are equivalent if and only if their zonal diagrams are equivalent.

We now return to the case of zonotopes which are the sums of line segments, two or more of which may be parallel. Let $Z=\left( \pm z_{1}, \ldots, \pm z_{n}\right)$, and suppose that $z_{n}$ (say) is a scalar multiple of some $z_{j}$ (with suffix $j \neq n$ ). Let $S_{i}=\operatorname{conv}\left\{-z_{i}, z_{i}\right\}$ $(i=1, \ldots, n)$. Then clearly the zonotopes

$$
P=S_{1}+\cdots+S_{n}, \quad P^{\prime}=S_{1}+\cdots+S_{n-1}
$$

are equivalent.

Now consider the central representation $\bar{Z}=\left( \pm \bar{z}_{1}, \ldots, \pm \bar{z}_{n}\right)$ of $Z$. The condition on $z_{n}$ is equivalent to the existence of an open half-space, bounded by a hyperplane through 0 which contains at most one point of $\bar{Z}$ apart from $\bar{z}_{n}$. From the results about central representations listed at the beginning of the section, if we project $\left( \pm \bar{z}_{1}, \ldots, \pm \bar{z}_{n-1}\right)$ orthogonally on to the hyperplane through 0 orthogonal to $\bar{z}_{n}$, we obtain a central representation of the subset $\left( \pm z_{1}, \ldots, \pm z_{n-1}\right)$.

We can clearly repeat this procedure, until finally we obtain a centrally symmetric set which corresponds to the distinct zones of the zonotope $P$; we shall call such a set a reduced diagram of $\bar{Z}$. It will generally be possible to obtain a reduced diagram of $\bar{Z}$ in many ways, since at each stage we may be presented with different choices of points $\bar{z}_{i}$ which lie in open half-spaces bounded by hyperplanes through 0 containing at most one point of $\bar{Z}$ apart from $\bar{z}_{i}$. However, consideration of the 
original zonotope $P$ shows that all these reduced diagrams are equivalent. We summarize this as follows.

THEOREM 3. Let

$$
P=S_{1}+\cdots+S_{n}, \quad S_{i}=\operatorname{conv}\left\{-z_{i}, z_{i}\right\} \quad(i=1, \ldots, n),
$$

be a zonotope, and let $\bar{Z}=\left( \pm \bar{z}_{1}, \ldots, \pm \bar{z}_{n}\right)$ be a central representation of $\left( \pm z_{1}, \ldots, \pm z_{n}\right)$. Then a zonal diagram of $P$ is equivalent to any reduced diagram of $\bar{Z}$.

Two results are immediate consequences of Theorem 3 and the preliminary remarks about central representations.

Proposition 4. With the notation of Theorem 3, a zonal diagram of $S_{1}+\cdots+S_{r}$ is equivalent to a reduced diagram of the orthogonal projection of $\left( \pm \bar{z}_{1}, \ldots, \pm \bar{z}_{r}\right)$ on to the subspace of $\operatorname{lin} \bar{Z}$ completely orthogonal to $\operatorname{lin}\left\{\bar{z}_{r+1}, \ldots, \bar{z}_{n}\right\}$.

Let us denote by $P /\left(S_{r+1}, \ldots, S_{n}\right)$ the zonotope obtained by projecting $P$ orthogonally on to the subspace of $E^{d}$ completely orthogonal to $\operatorname{lin}\left\{z_{r+1}, \ldots, z_{n}\right\}$. Then

Proposition 5. A zonal diagram of $P /\left(S_{r+1}, \ldots, S_{n}\right)$ is equivalent to a reduced diagram of $\left( \pm \bar{z}_{1}, \ldots, \pm \bar{z}_{r}\right)$.

Finally, let $\bar{X}=\left(\bar{x}_{1}, \ldots, \bar{x}_{m}\right)$ be a set of points in $E^{k}$ (say) such that

$$
0 \in \operatorname{int} \operatorname{conv} \bar{X} \text {. }
$$

So, by multiplying the points $\bar{x}_{i}$ by suitable positive numbers, we may assume that

$$
\bar{x}_{1}+\cdots+\bar{x}_{m}=0
$$

in which case $\bar{X}$ is an affine representation (or Gale transform, see Grünbaum [1967, 5.4]) of some set $X=\left(x_{1}, \ldots, x_{m}\right)$ in $E^{m-k-1}$. Not all points of $X$ will (in general) be vertices of the polytope $Q=\operatorname{conv} X$, so to find a Gale diagram of $Q$ we must apply the following reduction to $\bar{X}$, which is equivalent to eliminating those points $x \in X$ such that $x \in \operatorname{conv}(X \backslash\{x\})$. If $\bar{x} \in \bar{X}$ is such that there exists an open half-space of $E^{k}$ bounded by a hyperplane through 0 which meets $\bar{X}$ in $\bar{x}$ alone, then project $\bar{X} \backslash\{\bar{x}\}$ orthogonally on to the hyperplane through 0 orthogonal to $\bar{x}$. Repeat this process as often as possible (and if different choices of such $\bar{x}$ present themselves, perform the reduction with any such $\bar{x}$ ), finally obtaining a set of points $\bar{Y}$ in some subspace of $E^{k}$. We call $\bar{Y}$ a reduced diagram of $\bar{X} ; \bar{Y}$ will be a Gale diagram of $Q$.

It is known that, if $F$ is a $j$-face of a $d$-polytope $P$, then the sublattice of faces $F_{1}$ of $P$ such that $F \leqq F_{1} \leqq P$ forms a lattice which is isomorphic to the lattice of faces of some $(d-j-1)$-polytope, which we denote by $P / F$. (See Grünbaum [1967, 3.4, Exercise 10].) We then have the result analogous to McMullen-Shephard [1971, $\S 3$, Theorem 8]. 
Proposition 6. Let $F=S_{1}+\cdots+S_{r}+z_{r+1}+\cdots+z_{n}$ be a face of $P$. Then the reduced diagram of $\left(\bar{z}_{r+1}, \ldots, \bar{z}_{n}\right)$ is a Gale diagram of $P / F$.

4. Geometric formulations of zonal diagrams. In common with Gale diagrams and central diagrams, zonal diagrams can also be formulated geometrically. In this section we shall describe two closely related formulations, and show that they are equivalent to the algebraic formulation.

Let $P$ be a $d$-zonotope with $n$ zones. Keeping to the notation of the previous sections, its polar reciprocal is

$$
P^{*}=\left\{x \in E^{d}\left|\sum_{i=1}^{n}\right|\left\langle x, z_{i}\right\rangle \mid \leqq 1\right\} .
$$

Let $e_{1}, \ldots, e_{n}$ be linearly independent vectors in $E^{n}$, and let $X$ be the (not necessarily regular) cross-polytope with vertices $\pm e_{1}, \ldots, \pm e_{n}$. That is,

$$
X=\left\{y=\sum_{i=1}^{n} \eta_{i} e_{i}\left|\sum_{i=1}^{n}\right| \eta_{i} \mid \leqq 1\right\}
$$

We then have

THEOREM 4. Let $P^{*}$ and $X$ be as above. Then the map $\Lambda: E^{d} \rightarrow E^{n}$ defined by

$$
x \Lambda=\sum_{i=1}^{n}\left\langle x, z_{i}\right\rangle e_{i}
$$

is a one-to-one linear map such that

$$
P^{*} \Lambda=E^{d} \Lambda \cap X .
$$

The map $\Lambda$ is clearly linear, and it is one-to-one because $\left\{z_{1}, \ldots, z_{n}\right\}$ spans $E^{d}$. Further, the condition that $x \in P^{*}$ is $\sum_{i=1}^{n}\left|\left\langle x, z_{i}\right\rangle\right| \leqq 1$, which is also just the condition that $x \Lambda \in E^{d} \Lambda \cap X$. This proves the theorem.

We now describe the first geometric formulation. Let $M$ be the $(n-d)$-dimensional subspace of $E^{n}$ orthogonal to $L=E^{d} \Lambda$, and write $\Pi_{M}$ for orthogonal projection on to $M$. Then we define the set

$$
\bar{Z}=(\text { vert } X) \Pi_{M}
$$

to be a zonal diagram of $P$. If we write

$$
\bar{z}_{i}=e_{i} \Pi_{M} \quad(i=1, \ldots, n),
$$

then $\bar{z}_{i}$ is the point of $\bar{Z}$ corresponding to the point $z_{i}$. (We shall show in the course of the second geometric formulation that $\bar{Z}$ is also a zonal diagram in the algebraic sense.)

The zonal diagram condition of Theorem 1 can be deduced geometrically, as follows. Let

$$
F=S_{1}+\cdots+S_{r}+z_{r+1}+\cdots+z_{n}
$$


be a face of $P$. As we saw in $\S 1$, the corresponding face of $P^{*}$ is

$$
\hat{F}=\left\{x \in P^{*} \mid\left\langle x, z_{i}\right\rangle=0(i=1, \ldots, r),\left\langle x, z_{r+1}+\cdots+z_{n}\right\rangle=1\right\} .
$$

So, from the definition of the linear transformation $\Lambda$, we see that

$$
D=\operatorname{conv}\left\{e_{r+1}, \ldots, e_{n}\right\}
$$

is the (unique) face of $X$ of minimal dimension such that $\hat{F} \Lambda=L \cap D$; thus

$$
\text { rel int }(\hat{F} \Lambda) \subseteq \operatorname{rel} \text { int } D \text {. }
$$

Since $L \Pi_{M}=\{0\}$, and $\hat{F} \Lambda \subset L$, it follows that

$$
0 \in(\text { rel int } D) \Pi_{M}=\text { rel int conv }\left\{\bar{z}_{r+1}, \ldots, \bar{z}_{n}\right\} .
$$

The argument is completely reversible, and we thus obtain the condition of Theorem 1.

The geometrical formulation makes it easy to find the dimension of the face $F$ in terms of the zonal diagram $\bar{Z}$. For,

$$
\operatorname{dim} F+\operatorname{dim} \hat{F}=d-1 \text {, }
$$

and

$$
\operatorname{dim}(\hat{F} \Lambda)+\operatorname{dim} D \Pi_{M}=\operatorname{dim} D=n-r-1 .
$$

Since $\Lambda$ is one-to-one (and so $\operatorname{dim} \hat{F}=\operatorname{dim}(\hat{F} \Lambda)$ ), we deduce that

$$
\operatorname{dim} F=\operatorname{dim} \operatorname{lin}\left\{\bar{z}_{r+1}, \ldots, \bar{z}_{n}\right\}-n+d+r .
$$

Two special cases of this relation are of interest. Firstly, $F$ is a facet $(\operatorname{dim} F=d-1)$ if and only if

$$
\operatorname{dim} \operatorname{lin}\left\{\bar{z}_{r+1}, \ldots, \bar{z}_{n}\right\}=n-r-1 \text {; }
$$

that is, $\operatorname{conv}\left\{\bar{z}_{r+1}, \ldots, \bar{z}_{n}\right\}$ is a simplex with the origin in its relative interior. Secondly, $F$ is a cube (or, more properly, a paralleltope, so that $z_{1}, \ldots, z_{r}$ are linearly independent) if and only if

$$
\operatorname{dim} \operatorname{lin}\left\{\bar{z}_{r+1}, \ldots, \bar{z}_{n}\right\}=n-d ;
$$

that is, $\left\{\bar{z}_{r+1}, \ldots, \bar{z}_{n}\right\}$ spans $M$ positively.

The other geometric formulation is, in a sense, the dual of this, and is completely equivalent to it. However, the different formulation sheds new light on the relationship between a zonotope and its diagram, and enables us to relate the geometric and algebraic formulations.

It is clear that a zonotope with $n$ zones is the image under orthogonal projection of some (not necessarily regular) $n$-cube. For example, we may suppose that the zonotope $P$ lies in a $d$-dimensional (linear) subspace $L$ of $E^{n}$; we let $M$ denote the $(n-d)$-dimensional subspace of $E^{n}$ orthogonal to $L$. Using the same notation $P=S_{1}+\cdots+S_{n}, S_{i}=\operatorname{conv}\left\{-z_{i}, z_{i}\right\}(i=1, \ldots, n)$ as before, we may assume without loss of generality that $z_{1}, \ldots, z_{d}$ are linearly independent. Let $\left\{b_{d+1}, \ldots, b_{n}\right\}$ 
be a basis of $M$; then the points $z_{1}, \ldots, z_{d}, z_{d+1}+b_{d+1}, \ldots, z_{n}+b_{n}$ are clearly linearly independent points of $E^{n}$, whose orthogonal projections on to $L$ are $z_{1}, \ldots, z_{n}$. So, let $P$ be the orthogonal projection on to $L$ of the $n$-cube $C=T_{1}+\cdots+T_{n}, T_{i}=\operatorname{conv}\left\{-t_{i}, t_{i}\right\} \quad(i=1, \ldots, n)$, where $t_{1}, \ldots, t_{n}$ (which are linearly independent) are such that $z_{i}=t_{i} \Pi_{L}(i=1, \ldots, n)\left(\Pi_{L}\right.$ denoting the orthogonal projection on to $L$ ). Let

$$
X=\operatorname{conv}\left\{ \pm e_{1}, \ldots, \pm e_{n}\right\}
$$

be the cross-polytope which is the polar reciprocal of $C$, so that the vectors $t_{1}, \ldots, t_{n}$ and $e_{1}, \ldots, e_{n}$ satisfy the relations

$$
\left\langle t_{i}, e_{j}\right\rangle=\delta_{i j} \quad(i, j=1, \ldots, n),
$$

where $\delta_{i j}$ is the Kronecker delta function. Of course, the relationship between sections and projections implies that

$$
P^{*}=\left(C \Pi_{L}\right)^{*}=X \cap L,
$$

and so we see that (vert $X) \Pi_{M}$ is a zonal diagram of $P$, as previously formulated geometrically.

However, we are now in a position to recover the algebraic formulation. Let us write

$$
w_{i}=t_{i} \Pi_{M}, \quad \bar{z}_{i}=e_{i} \Pi_{M}, \quad \bar{w}_{i}=e_{i} \Pi_{L} \quad(i=1, \ldots, n)
$$

so that $\bar{Z}=\left( \pm \bar{z}_{1}, \ldots, \pm \bar{z}_{n}\right)$ is the zonal diagram of $P$. We may choose coordinates in $E^{n}$ so that the points of $L$ are those whose last $n-d$ coordinates vanish (and the points of $M$ are then those whose first $d$ coordinates vanish); we then write

$$
t_{i}=\left(z_{i}, w_{i}\right), \quad e_{i}=\left(\bar{w}_{i}, \bar{z}_{i}\right) \quad(i=1, \ldots, n)
$$

(rather than $t_{i}=z_{i}+w_{i}, e_{i}=\bar{w}_{i}+\bar{z}_{i}$ ). We further let

$$
T=\left(t_{1}^{T}, \ldots, t_{n}^{T}\right), \quad E=\left(e_{1}^{T}, \ldots, e_{n}^{T}\right)
$$

denote the matrices whose columns are the transposes of the vectors $t_{i}$ and $e_{i}$ (the superscript ${ }^{T}$ denotes transpose). The relation between the vectors $t_{i}$ and $e_{i}$ noted above is equivalent to $T^{T} E=I$, the $n \times n$ identity matrix. But then $E T^{T}=I$ also. Writing $\bar{z}_{i}=\left(\xi_{i 1}, \ldots, \xi_{i, n-d}\right) \quad(i=1, \ldots, n)$, we see that this gives at once $\sum_{i=1}^{n} \zeta_{i j} z_{i}=0(j=1, \ldots, n-d)$, which are precisely the defining relations for a zonal diagram of $P$ in the algebraic sense.

Finally, we can also obtain the zonal diagram condition using the geometric formulation of the zonal diagram by means of projections. For, let

$$
F=S_{1}+\cdots+S_{r}+z_{r+1}+\cdots+z_{n}
$$

be a face of $P$. Then $F=H \cap P$ for some supporting hyperplane

$$
H=\left\{x \in E^{d} \mid\langle x, u\rangle=1\right\}
$$


of $P$. But then $K=H \Pi_{L}^{-1}$ is a supporting hyperplane of $C$, such that

$$
K \cap C=T_{1}+\cdots+T_{r}+t_{r+1}+\cdots+t_{n} .
$$

Now

$$
K=\left\{y \in E^{n} \mid\langle y, u\rangle=1\right\},
$$

and these last two conditions imply that $u \in \operatorname{rel}$ int $D$, where $D=\operatorname{conv}\left\{e_{r+1}, \ldots, e_{n}\right\}$ is the face of the dual cross-polytope $X$ corresponding to $K \cap C$. As before, projecting orthogonally on to $M$ sends $u$ to 0 , and yields the zonal diagram condition. This argument is also completely reversible.

5. The derived zonotope. Let

$$
P=S_{1}+\cdots+S_{n}, \quad S_{i}=\operatorname{conv}\left\{-z_{i}, z_{i}\right\} \quad(i=1, \ldots, n),
$$

be a $d$-zonotope (where we again relax the condition that no two of the segments $S_{i}$ be parallel), and let

$$
\bar{Z}=\left( \pm \bar{z}_{1}, \ldots, \pm \bar{z}_{n}\right)
$$

be a central representation of $\left( \pm z_{1}, \ldots, \pm z_{n}\right)$. If we write

$$
\bar{S}_{i}=\operatorname{conv}\left\{-\bar{z}_{i}, \bar{z}_{i}\right\} \quad(i=1, \ldots, n)
$$

then

$$
\bar{P}=\bar{S}_{1}+\cdots+\bar{S}_{n}
$$

is an $(n-d)$-zonotope, which we shall call a zonotope derived from $P$. Clearly, $P$ is also a zonotope derived from $\bar{P}$. We remark that the derived zonotope $\bar{P}$ depends not on $P$ alone, but on the way of writing $P$ as a vector sum of line segments.

If we extend the definition of equivalence to sums of not necessarily nonparallel line segments in the obvious way, then (compare Proposition 3 ) if $Q$ is equivalent to $P$, the derived zonotope $\bar{Q}$ is equivalent to $\bar{P}$. However, we can obtain stronger results than this, and show that, in a certain sense, the correspondence between $P$ and the derived zonotope $\bar{P}$ is combinatorial in nature.

We first remark that Theorem 1 has the following consequence.

THEOREM 5. If

$$
F=S_{1}+\cdots+S_{r}+z_{r+1}+\cdots+z_{n}
$$

is a face of $P$, and

$$
z_{1}+\cdots+z_{n} \in \operatorname{rel} \text { int } F
$$

then

$$
\bar{F}=\bar{z}_{1}+\cdots+\bar{z}_{r}+\bar{S}_{r+1}+\cdots+\bar{S}_{n}
$$

is a face of $\bar{P}$, and

$$
\bar{z}_{1}+\cdots+\bar{z}_{n} \in \operatorname{rel} \text { int } \bar{F} \text {. }
$$


For, Theorem 1 implies that

$$
0 \in \text { rel int conv }\left\{\bar{z}_{r+1}, \ldots, \bar{z}_{n}\right\},
$$

which is the second conclusion. Further, the second hypothesis implies that

$$
0 \in \text { rel int conv }\left\{z_{1}, \ldots, z_{r}\right\} \text {, }
$$

and Theorem 1 gives the first conclusion immediately. This proves Theorem 5 .

COROLlaRY. For each choice $\varepsilon_{i}= \pm 1(i=1, \ldots, n), \varepsilon_{1} z_{1}+\cdots+\varepsilon_{n} z_{n}$ is a vertex (boundary point or interior point) of $P$ if and only if $\varepsilon_{1} \bar{z}_{1}+\cdots+\varepsilon_{n} \bar{z}_{n}$ is an interior point (boundary point or vertex) of $\bar{P}$.

The required combinatorial correspondence will then follow from

THEOREM 6. Suppose that $Z=\left( \pm z_{1}, \ldots, \pm z_{n}\right)$ is such that no hyperplane through 0 contains all of $z_{1}, \ldots, z_{n}$ but one, and that $z_{i} \neq 0(i=1, \ldots, n)$. Suppose further it is known for each $\left(\varepsilon_{1}, \ldots, \varepsilon_{n}\right)\left(\varepsilon_{i}= \pm 1, i=1, \ldots, n\right)$ whether the point $\varepsilon_{1} z_{1}+\cdots$ $+\varepsilon_{n} z_{n}$ is a vertex, boundary point or interior point of $P$. Then the combinatorial type of $P$ can be determined.

We observe firstly that the conditions on $Z$ ensure that $P$ has both vertices and interior points. The combinatorial type of $P$ is determined if we know which vertices of $P$ belong to each facet of $P$ (Grünbaum [1967, Exercise 3.2.3]), and so Theorem 6 follows immediately from the observation that the facets of $P$ are the subsets of the form

$$
F=S_{1 \sigma}+\cdots+S_{r \sigma}+\varepsilon_{r+1} z_{(r+1) \sigma}+\cdots+\varepsilon_{n} z_{n \sigma}
$$

which are maximal with respect to inclusion, such that every point

$$
\eta_{1} z_{1 \sigma}+\cdots+\eta_{r} z_{r \sigma}+\varepsilon_{r+1} z_{(r+1) \sigma}+\cdots+\varepsilon_{n} z_{n \sigma} \quad\left(\eta_{i}= \pm 1, i=1, \ldots, r\right)
$$

is a vertex or boundary point. This proves Theorem 6.

Recalling the correspondence of Proposition 1 between prismatic zonotopes and zero vectors in the zonal diagram, we see that if $Z$ satisfies the conditions of Theorem 6, so does its central representation $\bar{Z}$.

To obtain a correspondence between combinatorial types of zonotopes, we must impose further restrictions on the set $Z$. From now on, we shall assume that no two of the segments $S_{i}=\operatorname{conv}\left\{-z_{i}, z_{i}\right\}(i=1, \ldots, n)$ are parallel, so that $P$ is a zonotope with $n$ zones. To ensure that $\bar{P}$ is also a zonotope with $n$ zones, we must clearly demand that $Z$ satisfy the condition of Theorem 2 ; that is, no hyperplane through 0 can contain $n-2$ or more of $z_{1}, \ldots, z_{n}$. (Notice that we must have $d \geqq 2, n-d \geqq 2$ in this case.) Let us denote the class of $d$-zonotopes with this property $\mathscr{Z}_{*}^{d}$. We then deduce at once from the Corollary to Theorem 5 and Theorem 6

THEOREM 7. For each $d \geqq 2, n \geqq d+2$, there is a one-to-one correspondence between the combinatorial isomorphism classes of zonotopes in $\mathscr{Z}_{*}^{d}$ with $n$ zones and zonotopes in $\mathscr{Z}_{*}^{n-d}$ with $n$ zones. 
We can further refine this correspondence, as follows. We say a $d$-zonotope is cubical if all its proper faces are (combinatorially isomorphic to) cubes. In our usual notation, this clearly implies that no $d$ of the vectors $z_{1}, \ldots, z_{n}$ lie in a hyperplane through 0 ; that is, $\left\{z_{1}, \ldots, z_{n}\right\}$ is in linearly general position. It is easy to verify that the subset $\left\{\bar{z}_{1}, \ldots, \bar{z}_{n}\right\}$ of the zonal diagram must also be in linearly general position, so that the derived zonotope is also cubical. As before, we deduce from the Corollary to Theorem 5 and Theorem 6

THEOREM 8. For each $d \geqq 2, n \geqq d+2$, there is a one-to-one correspondence between the combinatorial types of cubical d-zonotopes with $n$ zones and cubical $(n-d)$-zonotopes with $n$ zones.

6. Enumeration of combinatorial types. In this section we shall consider the problem of enumerating the combinatorial types of zonotopes of a given dimension with a given number of zones.

We denote by $z(n, d)$ the number of combinatorial types of $d$-zonotopes with $n$ zones, and by $z_{c}(n, d)$ the number of combinatorial types of cubical $d$-zonotopes with $n$ zones. We first consider the case $n=d$. The zonal diagram of such a zonotope is 0 -dimensional, and so consists of $2 d$ points coincident at the origin. The combinatorial type is thus unique; it is that of the $d$-cube. So

TheOREM 9. For $d \geqq 1, z(d, d)=1=z_{c}(d, d)$.

We have remarked in Proposition 3 that equivalent zonotopes have equivalent zonal diagrams; thus, if we are concerned only with combinatorial type, we may replace the points $\bar{z}_{i}$ of the zonal diagram with any multiples of these by nonzero scalars. In particular, we may assume that, if $\bar{z}_{i} \neq 0$, then $\left\|\bar{z}_{i}\right\|=1$; we shall call such a zonal diagram standard.

In the case $n=d+1(d \geqq 2)$, the standard zonal diagram is 1-dimensional, and so consists of a centrally symmetric set of $2(d+1)$ points, each of which is \pm 1 or 0 . The zonal diagram condition of Theorem 2 implies that at least three points occur at +1 (and at -1 ). The zonotope is cubical if the points of the zonal diagram are in linearly general position; that is, none of them is zero. There is thus only one combinatorial type of cubical $d$-zonotope with $d+1$ zones. In the general case, if $2 k$ of the points of the zonal diagram occur at the origin, the corresponding zonotope is the $k$-fold prism whose basis is the cubical $(d-k)$-zonotope with $d-k+1$ zones. Summarizing:

THEOREM 10. If $d \geqq 2, z(d+1, d)=d-1, z_{c}(d+1, d)=1$.

We finally consider the case $n=d+2$. The standard zonal diagram is 2dimensional, and so is a subset of $S \cup\{0\}$, where $S$ is the unit circle in $E^{2}$. The nonzero points occur (in a centrally symmetric way) on at least two diameters of $S$. It is clear that we may rotate diameters of $S$ (and the points of the zonal diagram that they contain) in any fashion, as long as we do not allow two diameters to cross, without altering the combinatorial type of the corresponding zonotope. In 
particular, we may assume that the diameters of $S$ which contain points of the diagram are equally spaced. (Compare the corresponding result for Gale diagrams, Grünbaum $[1967,6.4]$.

For small numbers of diameters, the zonal diagram condition of Theorem 2 imposes certain restrictions. If there are two diameters, each must contain at least 3 pairs of nonzero points, and if there are three diameters, two of these must contain at least 2 pairs of nonzero points.

If the zonotope is cubical, there must be $d+2$ distinct diameters, which can only happen in one way. In the general case, from the discussion above we conclude that the number of combinatorial types of $d$-zonotopes with $d+2$ zones is the number of ways, distinct under orthogonal transformation, of distributing $2(d+2)$ points in a centrally symmetric manner at the vertices or centre of a regular $2 k$-gon $(k \geqq 2)$; if $k=2$, each vertex has at least 3 points, and if $k=3$, at least two pairs of opposite vertices have at least 2 points. This, in turn, is equal to the number of ways of distributing $d+2$ points at the vertices or centre of a regular $k$-gon; if $k=2$, each vertex has at least 3 points, and if $k=3$, at least two vertices have at least 2 points. This number may be calculated using Pólya's theorem (Pólya [1937]); we do not reproduce the details here, but state only the final result.

THEOREM 11. If $d \geqq 2, z_{c}(d+2, d)=1$,

$$
z(d+2, d)=\sum_{r=1}^{d+2} \frac{1}{2 r}\left\{\sum_{s \mid r} 2^{s} \varphi\left(\frac{r}{s}\right)\right\}-5 d-\frac{13}{2}+ \begin{cases}5.2^{d / 2}, & d \text { even, } \\ 7.2^{(d-1) / 2}, & d \text { odd },\end{cases}
$$

where $\varphi$ is Euler's function $(\varphi(t)$ is the number of positive integers less than, and prime to, $t$ ), and the inside sum is taken over all positive integer divisors $s$ of $r$.

7. Zonotopes and arrangements. A $k$-arrangement is a finite set of hyperplanes in real $k$-dimensional projective space, which do not contain a common point. The components of the complement of the hyperplanes are the interiors of convex $k$-polytopes; these polytopes, together with their faces, are called the faces of the arrangement. As with polytopes, we say that two arrangements are combinatorially isomorphic if there is a one-to-one inclusion preserving correspondence between their faces. (For more detailed information, consult Grünbaum [1967, Chapter 18].)

Our interest in arrangements is due to their connexion with zonotopes. For, bearing in mind the one-to-two correspondence between points of projective $k$ space and pairs of antipodal points of the $k$-sphere in $E^{k+1}$, we see that an arrangement corresponds to a set of hyperplanes through the origin of $E^{k+1}$ (which cut out a set of great spheres of the $k$-sphere), and so to the zonotope which is the vector sum of the diameters of the $k$-sphere orthogonal to the hyperplanes. From our description in $\$ 2$ of the dual of a zonotope, it follows that there is a two-toone correspondence between the faces of the dual and the faces of the original arrangement. 
In many cases it is more convenient to consider the analogous arrangements of points in projective $k$-space, not all of which lie in a hyperplane. The points of a point-arrangement correspond directly to diameters of the $k$-sphere, and so to zones of the associated zonotope. To the various subspaces spanned by subsets of points of the arrangement correspond sets of parallel faces of the zonotope.

Many results about zonotopes have been deduced from consideration of the corresponding arrangements (see, for example, Coxeter [1962] and Kelly-Moser [1958]), but there are few, if any, results in the other direction. Here, however, this situation can be remedied. Theorems 10 and 11 enable us to solve the problem of enumerating the combinatorial types of $k$-arrangements of $n$ hyperplanes, for $n \leqq k+3$. Since, in particular, to cubical zonotopes correspond simple arrangements, in which no more than $k$ hyperplanes pass through any point, we have the somewhat surprising

THEOREM 12. If $n \leqq k+3$, there is only one combinatorial type of simple $k$ arrangements of $n$ hyperplanes.

Furthermore, from Theorem 8 we at once deduce

THEOREM 13. If $k \geqq 1, n \geqq k+3$, there is a one-to-one correspondence between the combinatorial types of simple $k$-arrangements of $n$ hyperplanes and simple $(n-k-2)$ arrangements of $n$ hyperplanes.

Much of the interest in arrangements has centered around variants and extensions of Sylvester's problem (Sylvester [1893]). In an arrangement of points, we call a line ordinary if it contains exactly two of the points. Sylvester's question is equivalent to the following

Problem. Does every arrangement of points determine at least one ordinary line?

The question was first answered in the affirmative by Gallai (Grünwald) (see Erdös [1943]); Dirac [1951] showed that there are at least 3 ordinary lines, and Motzkin [1951] showed that the number of ordinary lines determined by $n$ points is at least $c \sqrt{ } n$, for some constant $c$. Kelly-Moser [1958] established the best known lower bound 3n/7; however, Motzkin [1951] has conjectured that the true lower-bound for the number of ordinary lines is $[n / 2]$, and examples show that no better bound is possible.

It should be remarked that Dirac's result can be obtained most easily by considering the 3-zonotope corresponding to the arrangement. We notice that ordinary lines correspond to pairs of parallel faces which are parallelograms. The faces of a 3-zonotope are polygons with an even number of sides; let $p_{2 i}$ denote the number of $2 i$-gonal faces, and $v_{j}$ the number of $j$-valent vertices. Then we easily deduce from Euler's equation and double counting of incidences (compare Grünbaum $[1967,13.1])$ the equation

$$
p_{4}=6+\sum_{i \geqq 4}(i-3) p_{2 i}+\sum_{j \geqq 4}(j-3) v_{j},
$$

from which we deduce at once that $p_{4} \geqq 6$. 
Among the extensions of Sylvester's problem that have been considered are those to higher dimensions. For example, we call a hyperplane spanned by points of a $k$-arrangement ordinary if all but one of the points of the arrangement in the hyperplane lie in a $(k-2)$-dimensional subspace. Motzkin [1951] established the existence of ordinary planes in 3-arrangements, and Hansen [1965] proved the same result for higher dimensions. The analogous result for zonotopes is

THEOREM 14. Every d-zonotope has at least one facet (or (d-1)-face) which is a prism.

Bonnice-Kelly [1970] have shown that a 3-arrangement of $n$ points determines at least $c n$ ordinary planes, where $c$ is some constant satisfying $3 / 11 \leqq c \leqq 2 / 3$.

We may use an easy induction argument to deduce from Theorem 14 the following result, which for arrangements gives a different generalization of Sylvester's problem.

THEOREM 15. Every $d$-zonotope has parallelotope $j$-faces, for each $j \leqq[(d+1) / 2]$.

If $n \geqq[3 d / 2]$, the example of the cartesian product of [d/2]-1 regular hexagons and, if $d$ is even a regular $(n-3[d / 2]+3)$-gon, or, if $d$ is odd any 3-zonotope with $n-3[d / 2]+3$ zones, shows that the result of Theorem 15 cannot be improved. However, for smaller $n$, we have the stronger

THEOREM 16. If $n \leqq[3 d / 2]$, a d-zonotope with $n$ zones has $j$-faces which are parallelotopes for each $j \leqq 2 d-n$.

For, consider the zonal diagram $\bar{Z}=\left( \pm \bar{z}_{1}, \ldots, \pm \bar{z}_{n}\right)$ of such a zonotope. The zonotope has (at least) one vertex, which we may assume to be $z_{1}+\cdots+z_{n}$. Then, by Theorem 1,

$$
0 \in \text { int } \operatorname{conv}\left\{\bar{z}_{1}, \ldots, \bar{z}_{n}\right\}
$$

that is, $\left\{\bar{z}_{1}, \ldots, \bar{z}_{n}\right\}$ spans $E^{n-d}$ positively. It follows by a theorem of Steinitz [1914] (see also Shephard [1970] for an elegant proof of this result) that some subset of at most $2(n-d)$ points of $\left\{\bar{z}_{1}, \ldots, \bar{z}_{n}\right\}$ spans $E^{n-d}$ positively. It follows that the zonotope has a face which is a parallelotope of dimension at least $n-2(n-d)$ $=2 d-n$, as claimed. This proves the theorem.

It is not hard to find examples to show that the result of Theorem 16 cannot be further improved.

The result on arrangements of points analogous to Theorem 16 is

THEOREM 17. For each $n \leqq[3(k+1) / 2]$ and $j \leqq 2 k+1-n$, a $k$-arrangement of $n$ points has $j$-dimensional subspaces spanned by and containing exactly $j+1$ points of the arrangement.

8. Concluding remarks. We have seen in the previous sections that the use of zonal diagrams sheds a considerable amount of light on the combinatorial structure 
of zonotopes, as well as leading to some new results on arrangements. It may be expected that other applications of zonal diagrams will be found in the future.

One possible avenue of investigation is suggested by the following. We say that a zonotope $P$ is zonally stable if every zonotope combinatorially isomorphic to $P$ is actually equivalent to $P$. A few obvious results present themselves: The cartesian product of zonally stable zonotopes is zonally stable; any $d$-zonotope with at most $d+1$ zones is zonally stable, and the zonally stable $d$-zonotopes with $d+2$ zones are precisely those whose zonal diagrams have at most 3 distinct diameters. The existence of an essentially irrational arrangement of 9 points in projective 2-space (Grünbaum [1967, 5.5]), which gives rise to a zonally stable 3-zonotope, shows that for each $d \geqq 3$, there exists a $d$-zonotope which cannot be realized, as a zonotope, so that all its vertices have rational coordinates. It would obviously be interesting to have a characterization of zonally stable zonotopes analogous to that of linearly stable polytopes (McMullen [1969]).

In a sense, Gale diagrams, central diagrams and zonal diagrams are very closely related. For example, a general polytope is a projection of a simplex, and dual to a section of a simplex, a centrally symmetric polytope is a projection of a crosspolytope, and dual to a section of a cube, and a zonotope is a projection of a cube, and dual to a section of a cross-polytope. These representations lead to completely analogous definitions of the corresponding diagrams. Further, such analogies seem to lead one to the conclusion that there are no more diagram techniques for polytopes apart from these three.

However, since this work was done, Shephard [1970] has shown that a similar diagram technique, that of positive diagrams, can be applied to investigate positive bases of $E^{d}$. The use of positive diagrams, which can be regarded as inverses of Gale transforms (Grünbaum [1967, 5.4]), leads to more intuitive and perspicuous proofs of many results on positive bases, including the theorem of Steinitz [1914] mentioned in $\S 7$.

\section{REFERENCES}

Where possible, reference is made to a review of the paper concerned in Mathematical Reviews (MR), Zentralblatt für Mathematik (Z), or Jahrbuch über die Fortschritte der Mathematik (FM). A paper yet to be published is, in the text, arbitrarily assigned the date [1970].

W. E. Bonnice and L. M. Kelly, On the number of ordinary planes, J. Combinatorial Theory (to appear).

H. S. M. Coxeter, The classification of zonohedra by means of projective diagrams, J. Math. Pures Appl. (9) 41 (1962), 137-156. MR 25 \#4417.

G. A. Dirac, Collinearity properties of sets of points, Quart. J. Math. Oxford Ser. (2) 2 (1951), 221-227. MR 13, 270.

P. Erdös, Problem no. 4065, Amer. Math. Monthly 50 (1943), 65.

D. Gale, Neighboring vertices on a convex polyhedron. Linear inequalities and related systems, Ann. of Math. Studies, no. 38, Princeton Univ. Press, Princeton, N. J., (1956), pp. 255-263. MR 19, 57. 
B. Grünbaum, Convex polytopes, Pure and Appl. Math., vol. 16, Interscience, New York, 1967. MR 37 \#2085.

S. Hansen, A generalization of a theorem of Sylvester on the lines determined by a finite point set, Math. Scand. 16 (1965), 175-180. MR 34 \#3411.

L. M. Kelly and W. O. J. Moser, On the number of ordinary lines determined by $n$ points, Canad. J. Math. 1 (1958), 210-219. MR 20 \#3494.

P. McMullen, Linearly stable polytopes, Canad. J. Math. 21 (1969), 1427-1431.

P. McMullen and G. C. Shephard, Diagrams for centrally symmetric polytopes, Mathematika 15 (1968), 123-138. MR 38 \#6456.

- Convex polytopes and the upper-bound conjecture, London Math. Soc. Lecture Note Series, vol. 3, 1971.

T. S. Motzkin, The lines and planes connecting the points of a finite set, Trans. Amer. Math. Soc. 70 (1951), 451-464. MR 12, 849.

G. Pólya, Kombinatorische Anzahlbestimmungen für Gruppen, Graphen und chemische Verbindungen, Acta Math. 68 (1937), 145-254, 217, 232.

G. C. Shephard, Diagrams for positive bases, J. London Math. Soc. (to appear).

E. Steinitz, Bedingt konvergente Reihen und konvexe Systeme. II, J. Reine Angew. Math. 144 (1914), 1-40. FM 44, 287.

J. J. Sylvester, Mathematical question 11851, Ed. Times 59 (1893), 98.

Michigan State University,

East Lansing, Michigan 48823

UNIVERSITY COLlege,

LONDON, ENGLAND 